\title{
Effects of the vasodilating beta-blocker nebivolol on smoking-induced endothelial dysfunction in young healthy volunteers
}

\author{
André C Schmidt ${ }^{1}$ \\ Burkhard Flick' \\ Elke Jahn² \\ Peter Bramlage ${ }^{3}$ \\ 'Charité - Universitätsmedizin Berlin, \\ Institute for Clinical Pharmacology \\ and Toxikology, Berlin, Germany; \\ ${ }^{2}$ Berlin-Chemie AG, Clinical Research \\ and Medical Information, Berlin, \\ Germany; ${ }^{3}$ Institute for Clinical \\ Pharmacology, Medical Faculty Carl \\ Gustav Carus, TU Dresden, Germany
}

Correspondence:André C Schmidt Charité Universitätsmedizin Berlin, Institute for Clinical Pharmacology and Toxikology, Hindenburgdamm 30, 12200 Berlin, Germany

$\mathrm{Tel}+49$ I 633106434

Fax +4933708928069

Email andre.schmidt@charite.de
Objective: To assess the effect of nebivolol, a highly selective third generation $\beta_{1}$-adrenoceptor antagonist with an endothelium-dependent vasodilatory action, on smoking-induced endothelial dysfunction.

Research design and methods: This open-label study examined the effect of 14 daily doses of $5 \mathrm{mg}$ nebivolol on forearm blood flow in 21 healthy, young, male, light smokers ( $\leq 5$ cigarettes/day), measured by plethysmography on Days 1,7 , and 14 . The primary endpoint was the difference in forearm blood flow after smoking one standard cigarette from baseline (Day 1) until treatment end on Day 14. Secondary outcomes included the difference in forearm blood flow between Day 1 and Day 7 compared with Day 14 before and after smoking, the effect of nebivolol on blood coagulation parameters, high-sensitive-C-reactive protein (hs-CRP), and the safety and tolerability of nebivolol.

Results: Nebivolol for 14 days did not significantly affect forearm blood flow after smoking. On Day 7 of nebivolol treatment, forearm blood flow after smoking was significantly greater than blood flow before smoking (increase of $0.44 \mathrm{~mL} / \mathrm{min} ; \mathrm{p}=0.00656$ ). Serum level of hs-CRP showed a marked decrease from Day 1 to Day 14. No changes in coagulation parameters were observed over the course of nebivolol treatment. Nebivolol was well tolerated throughout the study.

Conclusions: The increase in forearm blood flow and the marked decrease in hs-CRP over 14 days of treatment suggest that nebivolol has a positive effect on endothelial function in light smokers, but larger studies are required to confirm these observations.

Keywords: C-reactive protein, endothelial dysfunction, nebivolol, nitric oxide (NO), smoking

\section{Introduction}

The endothelium is responsible for the synthesis of the potent vasodilator nitric oxide (NO). $\mathrm{NO}$ is involved in a large number of cardiovascular processes, and NO deficiency represents an important determinant of cardiovascular risk (Moncada and Higgs 2006). Endothelial dysfunction and the associated reduced synthesis or action of NO may lead to vasoconstriction, elevated blood pressure and thrombus formation, and can ultimately result in atherosclerosis (Brunner et al 2005; Moncada and Higgs 2006).

Cigarette smoking is a major modifiable risk factor for atherosclerosis and peripheral artery disease (Lu and Creager 2004). The pathophysiology of these potentially fatal diseases has been linked, at least in part, to cigarette-induced increases in oxidative stress. By enhancing the production of superoxide and other reactive oxygen species, cigarette smoke inactivates $\mathrm{NO}$ within the vasculature, thereby disrupting endothelial function (Cai and Harrison 2000). Indeed, long-term smokers show markedly impaired endothelium-dependent vasodilation, demonstrated in, for example, the brachial artery, 
which is consistent with endothelial dysfunction (Celermajer et al 1993). Smoking cessation has been shown to drastically improve endothelial function by limiting vascular endothelial damage as well as improving lipid profile and decreasing thrombotic tendency (Eagles and Martin 1998).

Smoking is also associated with increased levels of C-reactive protein (CRP) (Kao et al 2006). Recent research has confirmed a role for CRP in cardiovascular risk prediction (Willerson and Ridker 2004), and CRP has also been directly been involved in endothelial dysfunction in experimental models (Wilson et al 2006). Serum CRP level, as well as representing a marker of inflammation, is also, therefore, a valid means of quantifying endothelial dysfunction.

Nebivolol is the newest third-generation cardioselective beta-blocker with vasodilator activity. In addition to its conventional antihypertensive effect, nebivolol also has an endothelium-dependent vasodilatory action that may slow or prevent some of the vascular complications associated with hypertension, and may be of particular benefit in patients with impaired endothelial function (eg, diabetes, hypercholesterolemia, or ischemic heart disease) (Cockcroft 2004). A recent study showed that the treatment of hypertensive patients, concomitantly suffering from type-2 diabetes, with nebivolol not only reduced systolic and diastolic blood pressure, but also improved metabolic parameters (eg, HbA1c) and physical capability of those patients (Schmidt et al 2007).

The vasodilatory effect of nebivolol is thought to derive from its ability to stimulate NO production by endothelial cells (Broeders et al 2000). Infusion of nebivolol into the brachial artery has been shown to increase forearm blood flow in normotensive individuals, and this effect, which is antagonized by the NO synthase inhibitor NG-monomethylL-arginine (L-NMMA), implicates the L-arginine-NO pathway in nebivolol-induced vasodilation (Cockcroft et al 1995). By preserving endothelial function, nebivolol may help to reduce peripheral resistance and arterial stiffness, and may ultimately protect against cardiovascular disease (Luscher et al 2001).

This open-label pilot study examined the effects of a therapeutic dose of nebivolol on forearm blood flow, measured by plethysmography, and level of inflammatory marker, in light smokers over a 14-day period.

\section{Methods}

\section{Study design}

This was an open-label, single-center study that examined the efficacy and safety of oral nebivolol $5 \mathrm{mg}$ given once daily (Figure 1). It was conducted according to the Declaration of
Helsinki, taking into account the recent version of the German Drug Law (AMG), and in accordance with the German legal requirements as well as the principles of Good Clinical Practice (GCP). Blood and urine samples were taken on Day 1, in overnight-fasted healthy voluteers. Subjects then received a standard breakfast and forearm plethysmography was performed 2 hours after breakfast. Vital signs and 12-lead ECG measurements were taken after plethysmography. Each subject then received nebivolol $5 \mathrm{mg}$ (about 3 hours after breakfast). Further visits to the study center were scheduled on Days 7 and 14 for plethysmography and other efficacy and safety evaluations, as on Day 1. On Days 2-14, nebivolol $5 \mathrm{mg}$ was taken at home with breakfast, with the exception of days 7 and 14, when nebivolol was taken with breakfast at the study center.

Adverse events were recorded by subjects in diaries. A final follow-up examination took place within 8 days of the last nebivolol dose.

\section{Study population}

Healthy male subjects aged 18-35 years, all of whom were light smokers ( $\leq 5$ cigarettes/day), were eligible. Written informed consent was obtained from all subjects prior to any study related procedure. All subjects underwent a physical examination up to 7 days prior to enrollment (full medical history, 12-lead electrocardiography [ECG], blood pressure, heart rate, height and weight, lung function, hematology, and urinalysis) and were excluded from the study if they exhibited any clinically relevant abnormality. Subjects with concomitant illness were excluded from the study, as were those subjects with a history of smoking more than 5 cigarettes per day or who were judged to be heavy smokers according to clinical assessment. Subjects with a forced expiratory volume in 1 minute as a percentage of forced vital capacity $>70 \%$ (Tiffenau-Test) at screening were excluded. Subjects were also excluded if they were allergic to or had contraindications to the study drug or beta-blockers in general, were using any prescription or nonprescription drugs, or had a history of drug or alcohol abuse.

\section{Study measures}

The primary endpoint of this study was the difference in forearm blood flow between Day 1 and Day 14 after smoking. Secondary endpoints included forearm blood flow differences between Days 1 and 7, and Days 1 and 14, changes in coagulation parameters and high-sensitive-C-reactive protein (hs-CRP), as well as safety and tolerability of nebivolol in the study population. 


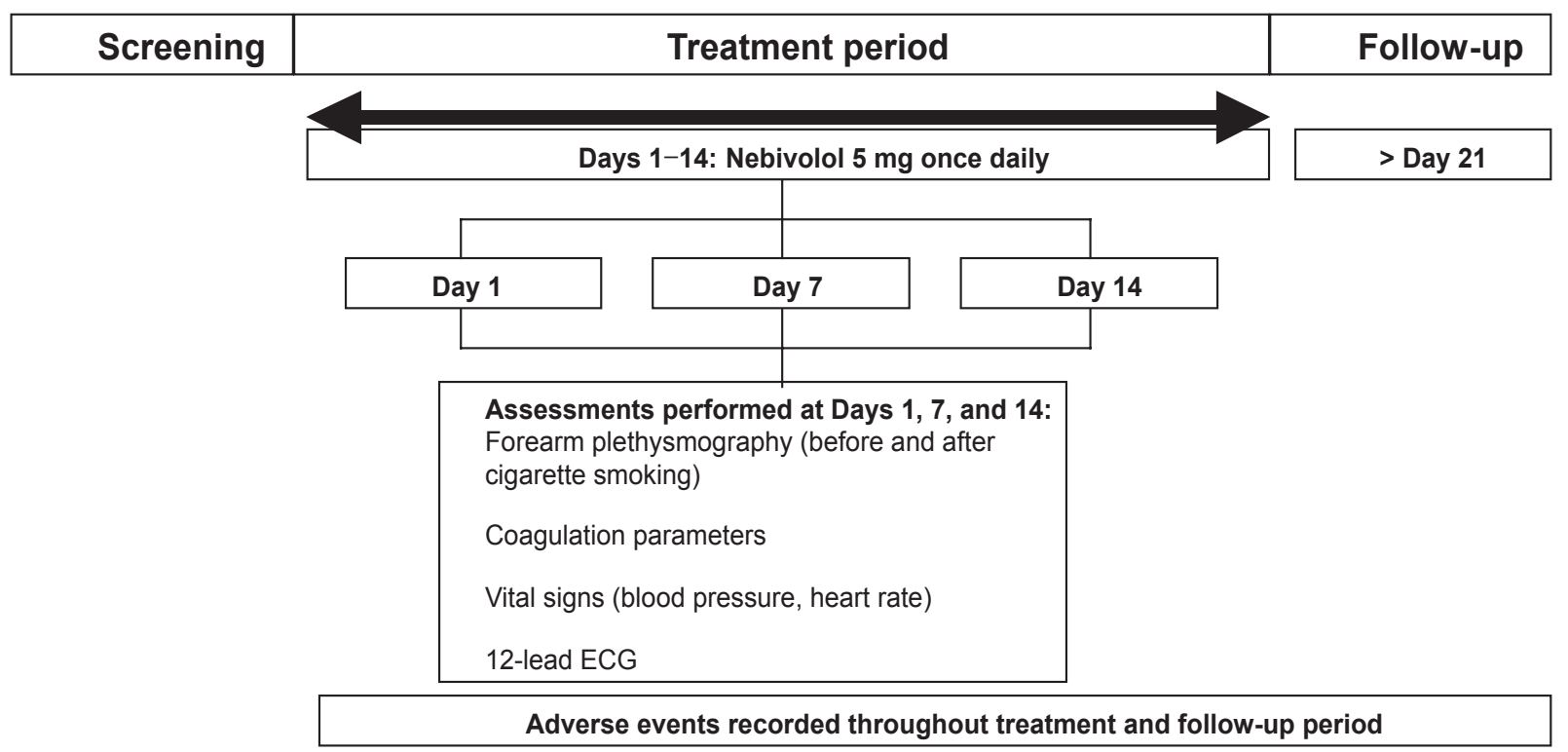

Primary endpoint:

Secondary endpoints:

Safety analysis:
Outcome measures

Difference in forearm blood flow between Day 1 and Day 14 after smoking.

Difference in forearm blood flow between Day 1, Day 7, and Day 14 before and after smoking; changes in coagulation parameters.

Vital signs, routine laboratory testing, ECG recording, reporting of adverse events,

Figure I Study protocol.

\section{Forearm plethysmography}

Plethysmography recordings (as a surrogate measure of endothelial function) were made twice during each outpatient visit, before and after smoking a cigarette (Marlboro ${ }^{\circledR}$ regular strength). The subject rested for 20 minutes in a supine position before and after the first recording. During the last 10 minutes of the rest period after the first recording, each subject smoked a cigarette and inhaled the smoke. Postsmoking forearm plethysmography was initiated $15 \pm 5$ minutes after the subject had finished smoking the cigarette. Forearm plethysmography was performed in the dominant arm according to standard methodology (Whitney 1953; Roddie and Wallace 1979). Forearm blood flow was recorded over a series of 3-minute measurement periods. Recordings taken during the first minute after wrist cuff inflation were discarded because of the reflex vasoconstriction, and the last 5 flow recordings of the 3 -minute measurement period were averaged. The rate of swelling of the forearm was calculated from changes of forearm circumference by means of a strain gauge placed around the forearm approximately a third of the way below the elbow.

\section{Vital signs and ECG}

Vital signs (including blood pressure and heart rate) and ECG were recorded on Days 1, 7, and 14. Blood pressure was measured in a sitting position after 10 minutes of rest. Three measurements were taken, with 2 minutes between each measurement and the mean value of the last 2 measurements at each visit was recorded.

\section{Blood parameters}

Blood samples $(3 \mathrm{~mL})$ were taken, placed on ice, and shipped to a central laboratory for analysis. Antithrombin III, fibrinogen and serum level of hs-CRP were determined using Sarstedt Citrate Monovettes ${ }^{\circledR}$.

\section{Statistical analysis}

The change in forearm blood flow between Days 1 and 14, as measured by forearm plethysmography after smoking (the primary endpoint), was analyzed by a paired signed rank test. As the present study was planned and designed as a pilot study, all other efficacy and safety data were analyzed descriptively and were not subjected to formal statistical 
analysis. The primary population for analysis included all subjects who received study medication, and who were evaluated at a minimum of 2 visits. The safety analysis included all subjects who had received at least 1 dose of study medication.

\section{Results}

Twenty-one subjects were enrolled in the study and 20 completed the 14-day treatment period. The mean age of the study population was 26.2 years, with a body weight average of $81.1 \mathrm{~kg}$ and a body mass index of $24.8 \mathrm{~kg} / \mathrm{m}^{2}$. One subject withdrew from the study on Day 12 after developing influenza; data from this subject up to Day 7 were included in the primary and secondary efficacy and safety evaluations. Another subject developed a sore throat on Day 13 of treatment, and as a result, registered high hs-CRP levels at Day 14 (47.20 mg/L). Neither of the subject withdrawals was considered to be related to the study medication.

\section{Forearm plethysmography}

The nebivolol-induced change in forearm blood flow after smoking between Days 1 and 14 was not significant (Table 1). No statistically significant nebivolol-induced changes in forearm blood flow were detected between any of the test days, either before or after smoking a cigarette (Table 1). On Day 7 of nebivolol treatment, forearm blood flow after smoking was significantly greater than blood flow before smoking (increase of $0.44 \mathrm{~mL} / \mathrm{min} ; \mathrm{p}=0.00656$; Table 2).

\section{Vital signs and ECG}

Mean seated systolic blood pressure (SBP) and diastolic blood pressure (DBP) decreased slightly between Day 1 and Day 14 (reductions of 3.8 and $2.7 \mathrm{mmHg}$, respectively). Heart rate also decreased from $64.4 \mathrm{bpm}$ on Day 1 to $60.9 \mathrm{bpm}$ on Day 14. No clinically significant ECG changes were found for any of the subjects.

Table I Summary of forearm blood flow changes between Days I, 7, and 14, before and after smoking I cigarette

\begin{tabular}{lllll}
\hline & \multicolumn{4}{c}{ Change in blood flow (mL/min) } \\
\hline & Change & N & Mean & SD \\
\hline Before smoking & Day 14-Day I & 20 & -0.09 & 1.01 \\
& Day 7-Day I & 21 & -0.33 & 0.80 \\
& Day 14-Day 7 & 20 & 0.25 & 0.75 \\
After smoking & Day 14-Day I & 20 & -0.07 & 1.22 \\
& Day 7-Day I & 21 & 0.08 & 1.23 \\
& Day 14-Day 7 & 20 & -0.18 & 1.39 \\
\hline
\end{tabular}

Table 2 Summary of forearm blood flow before and after smoking I cigarette on Days I, 7, and I4

\begin{tabular}{lllll}
\hline & & \multicolumn{3}{l}{ Blood flow (mL/min) } \\
\hline Day I & Before smoking & N & Mean & SD \\
& After smoking & 21 & 1.42 & 0.90 \\
& Change & & 1.45 & 1.17 \\
Day 7 & Before smoking & 21 & $\mathbf{0 . 0 3}$ & $\mathbf{0 . 6 3}$ \\
& After smoking & 21 & 1.09 & 0.53 \\
& Change & & 1.53 & 1.23 \\
Day 14 & Before smoking & 20 & $\mathbf{0 . 4 4} *$ & $\mathbf{0 . 9 1}$ \\
& After smoking & 20 & 1.37 & 0.74 \\
& Change & & 1.41 & 0.82 \\
& & & $\mathbf{0 . 0 3}$ & $\mathbf{0 . 2 9}$ \\
\hline
\end{tabular}

${ }^{*} p=0.00656$

\section{Blood parameters}

Serum levels of hs-CRP decreased markedly between Day 1 and Day 14, but there was a high variability among subjects (Table 3). No changes were reported in antithrombin III levels between Days 1 and 14 (Table 3). Some subjects showed pathological antithrombin III values but these were attributed to changes in sampling conditions. Fibrinogen values did not change between Days 1 and 14.

\section{Safety}

Nebivolol was generally well tolerated. In total, 15 adverse events were reported in 9 of the 21 subjects. Adverse events were mild (13 adverse events [86.6\%]) or moderate ( 2 adverse events [13.3\%]). Of the 15 adverse events, 9 were considered to be possibly related to the study medication. The most frequent adverse event, tiredness, was reported on 4 occasions. All other adverse events occurred only once and included nausea, asthenia, influenza, nasopharyngitis, muscle cramps, headache, and sore throat. No deaths or serious adverse events were reported and all adverse events resolved completely.

\section{Discussion}

This study is the first, to our knowledge, to investigate the effects of nebivolol on forearm blood flow in young, normotensive light smokers. Forearm blood flow, assessed by plethysmography, was used as a surrogate marker of endothelial dysfunction (Luscher and Noll 1996). The results revealed no change in endothelial function between Day 1 and Day 14. However, between Day 1 and Day 7 of nebivolol treatment there was a significant increase in forearm blood flow. There was also no difference in endothelial function before or after smoking 1 cigarette, either on the test day (with the exception of Day 7) or between 3 consecutive test days 
Table 3 Laboratory data efficacy on Days I and I4

\begin{tabular}{lllll}
\hline & & N & Mean & SD \\
\hline Antithrombin III (\%) & Day I & 20 & 94.62 & II.49 \\
& Day I4 & 19 & 98.78 & 12.88 \\
& Change & & $\mathbf{3 . 8 3}$ & 16.33 \\
Fibrinogen (G/L) & Day I & 20 & 2.79 & 0.49 \\
& Day I4 & 19 & 2.57 & 0.40 \\
& Change & & $-\mathbf{0 . 2 5}$ & $\mathbf{0 . 5 2}$ \\
hs-CRP (mg/L) & Day I & 19 & 2.05 & 1.96 \\
& Day I4 & 19 & 1.14 & 1.51 \\
& Change & & -1.04 & $\mathbf{2 . 5 8}$ \\
\hline
\end{tabular}

Abbreviation: hs-CRP, high-sensitive-C-reactive protein.

(Days 1, 7, and 14). Nebivolol was well tolerated throughout the study and exhibited a safety profile consistent with clinical experience. The slight decrease in blood pressure observed over the course of the study is consistent with the known antihypertensive profile of nebivolol.

The mechanisms of endothelial dysfunction after smoking are complex and heterogeneous (Brunner et al 2005). A number of previous studies have linked long-term smoking (up to 75 pack years over an individual's lifetime) with endothelial dysfunction - as detected by high-resolution ultrasound and physiochemical parameters - which is likely to relate to cigarette-induced detrimental effects on flowmediated dilation of arteries and the disruption of vessel wall morphology (Celermajer et al 1993; Esen et al 2004). Cigarette smoke extract has been shown to inhibit the uptake of arginine, reduce activity of endothelial NO synthase, elevate oxidative stress, and increase levels of asymmetric dimethylarginine (ADMA) (Zhang et al 2006). High plasma concentrations of ADMA are associated with intima-media thickening, left ventricular hypertrophy, and all-cause and cardiovascular mortality in patients with end-stage renal disease, and to coronary events in males in the general population (Zoccali 2006).

Nebivolol is known to relax vascular smooth muscle by a mechanism thought to involve stimulation of endothelial NO synthase-dependent NO production, which is induced by a $\beta_{2}$-adrenergic receptor-mediated rise in endothelial $\mathrm{Ca}^{2+}$ (Broeders et al 2000). A study in normotensive individuals showed that the infusion of nebivolol into the brachial artery increased forearm blood flow by $91 \%(\mathrm{p}<0.01)$ (Cockcroft et al 1995). L-NMMA reduced nebivolol-induced vasodilation by $62 \%$, an effect that was abolished by the NO precursor, L-arginine, thereby clearly implicating the L-arginine-NO pathway in nebivolol-induced vasodilation (Cockcroft et al 1995). It should also be noted that the effect of nebivolol on flow-mediated dilation of the brachial artery is unique to nebivolol and is not a class effect that is shared with other beta-blockers. In patients with coronary artery disease, flowmediated dilation of the brachial artery was unchanged after 4 weeks' treatment with the beta-blocker atenolol, but was increased significantly in patients treated with nebivolol (3.9\% versus $5.6 \% ; p=0.041$ ) (Lekakis et al 2005).

The current short-term study shows modest nebivololinduced increases in forearm blood flow after smoking at all time points measured. This finding may derive from the aforementioned ability of nebivolol to stimulate the release of NO from endothelial cells and, thereby, elicit vasodilation. Why the effect of nebivolol is only significant after smoking on Day 7 is unclear, but may be attributable to a high degree of variability in a small study population. In the current study, the difference in forearm blood flow preand postsmoking was for the most part negligible. Previous studies have shown that the smoking-induced attenuation in endothelium-dependent vasodilation is maximal shortly after initiation of smoking and persists for between 30 and 50 minutes (Sarabi and Lind 2000). The apparent failure to detect significant changes in forearm blood flow pre- and postsmoking in the current study may be attributable to the delay in initiating plethysmography (up to 20 minutes after smoking). Performing the procedure at a shorter time interval postsmoking may be more conducive to detecting maximal effects and is worth further investigation.

Previous studies have shown that endothelial dysfunction in smokers is directly proportional to the lifetime dose smoked and is largely reversible in asymptomatic young smokers (Celermajer et al 1993; Lind et al 2003). Celermajer et al (1993) found that flow-mediated dilation of the brachial artery in smokers was inversely related to lifetime dose smoked $(6.6 \%, 4.0 \%, 3.2 \%$, and $2.6 \%$ for very light, light, moderate, and heavy smokers respectively; $p<0.01$ ). Lind et al (2003) examined endothelium-dependent vasodilation, measured by forearm plethysmography in 2 groups of apparently healthy subjects with mean ages of 50 and 25 years. In the younger subjects, no differences in forearm vasodilation were observed between smokers and nonsmokers. In the population as a whole, however, endothelium-dependent vasodilation showed a significant inverse relationship with duration of smoking $(\mathrm{r}=-0.52, \mathrm{p}<0.05)$.

On the basis of these findings, the young, male, light smokers in the current study were unlikely to have chronically impaired endothelial function. It is conceivable that the vasodilatory effects of nebivolol may be dependent on the extent of underlying endothelial dysfunction. Examining the effects of nebivolol in an older population of smokers 
with a more extensive smoking history (and thus greater impairment of endothelial function) may reveal more pronounced effects of nebivolol. The absence of any significant changes in thrombotic activity in this study may also be attributed to patient population and the duration of the study.

Elevated CRP levels are associated with blunted systemic endothelial vasodilator function that is indicative of a systemic inflammatory response (Fichtlscherer and Zeiher 2000). Acute infection is also known to elevate levels of CRP (Ablij and Meinders 2002). The decision to exclude data from the subjects in the current study who developed influenza and a sore throat is justified on the basis that inflammatory state determined by viral agents may transitorily alter endothelial function in healthy subjects (Marchesi et al 2005). Normal values for CRP range between 0 and $10 \mathrm{mg} / \mathrm{L}$ and the inclusion of individual patient data approximately 5 times outside the normal range may have led to misinterpretation of the CRP result had they been included.

Smoking is known to increase serum CRP levels (Kao et al 2006) and, indeed, smoking cessation is recommended for those patients with elevated CRP levels (Blake and Ridker 2002). The significance of the current marked reduction in inflammatory markers following treatment with nebivolol is consistent with its known role as a vasodilator and is of potential clinical importance, particularly as CRP emerges as a therapeutic target in the management of cardiovascular disease processes (Verma et al 2005).

\section{Conclusions}

In summary, the slightly increased forearm blood flow after nebivolol treatment at Day 7 and the reduced levels of hs-CRP as a measure of inflammation suggest that nebivolol may have a positive effect on the endothelial function of light smokers.

Ultimately, this finding may have important implications regarding the selection of antihypertensives on the basis of smoking status. An existing study has shown that the effects of nebivolol on endothelial function and the prothrombotic state were more pronounced in hypertensive smokers than in hypertensive nonsmokers (as measured by decreases in fibrinogen, plasminogen activator inhibitor-I, and homocysteine [Vyssoulis et al 2004]). The failure of the beta-blockers, celiprolol and carvedilol, to elicit such pronounced effects in the same study demonstrates that the finding was unique to nebivolol and was not a class effect.

The limited size of the current study limits any firm conclusions, but further investigations in a larger and broader population are warranted.

\section{Acknowledgments}

The study was supported by BERLIN-CHEMIE AG, Berlin, Germany.

\section{Disclosures}

None of the authors has any conflicts of interest to disclose.

\section{References}

Ablij H, Meinders A. 2002. C-reactive protein: history and revival. Eur J Intern Med, 13:412.

Blake GJ, Ridker PM. 2002. Inflammatory bio-markers and cardiovascular risk prediction. J Intern Med, 252:283-94.

Broeders MA, Doevendans PA, Bekkers BC, et al. 2000. Nebivolol: a thirdgeneration beta-blocker that augments vascular nitric oxide release: endothelial beta2-adrenergic receptor-mediated nitric oxide production. Circulation, 102:677-84.

Brunner H, Cockcroft JR, Deanfield J, et al. 2005. Endothelial function and dysfunction. Part II: Association with cardiovascular risk factors and diseases. A statement by the Working Group on Endothelins and Endothelial Factors of the European Society of Hypertension. J Hypertens, 23:233-46.

Cai H, Harrison DG. 2000. Endothelial dysfunction in cardiovascular diseases: the role of oxidant stress. Circ Res, 87:840-4.

Celermajer DS, Sorensen KE, Georgakopoulos D, et al. 1993. Cigarette smoking is associated with dose-related and potentially reversible impairment of endothelium-dependent dilation in healthy young adults. Circulation, 88:2149-55.

Cockcroft J. 2004. Nebivolol: a review. Expert Opin Pharmacother, 5:893-9.

Cockcroft JR, Chowienczyk PJ, Brett SE, et al. 1995. Nebivolol vasodilates human forearm vasculature: evidence for an L-arginine/NO-dependent mechanism. J Pharmacol Exp Ther, 274:1067-71.

Eagles CJ, Martin U. 1998. Non-pharmacological modification of cardiac risk factors: part 3. Smoking cessation and alcohol consumption. J Clin Pharm Ther, 23:1-9.

Esen AM, Barutcu I, Acar M, et al. 2004. Effect of smoking on endothelial function and wall thickness of brachial artery. Circ J, 68:1123-6.

Fichtlscherer S, Zeiher AM. 2000. Endothelial dysfunction in acute coronary syndromes: association with elevated C-reactive protein levels. Ann Med, 32:515-8.

Kao PC, Shiesh SC, Wu TJ. 2006. Serum C-reactive protein as a marker for wellness assessment. Ann Clin Lab Sci, 36:163-9.

Lekakis JP, Protogerou A, Papamichael C, et al. 2005. Effect of nebivolol and atenolol on brachial artery flow-mediated vasodilation in patients with coronary artery disease. Cardiovasc Drugs Ther, 19:277-81.

Lind L, Sarabi M, Millgard J. 2003. The effect of smoking on endothelial vasodilatory function evaluated by local infusion of metacholine in the forearm is dependent on the duration of smoking. Nicotine Tob Res, 5:125-30.

Lu JT, Creager MA. 2004. The relationship of cigarette smoking to peripheral arterial disease. Rev Cardiovasc Med, 5:189-93.

Luscher TF, Noll G. 1996. Endothelial function as an end-point in interventional trials: concepts, methods and current data. J Hypertens Suppl, 14:S111-9; discussion S119-21.

Luscher TF, Spieker LE, Noll G, et al. 2001. Vascular effects of newer cardiovascular drugs: focus on nebivolol and ACE-inhibitors. J Cardiovasc Pharmacol, 38(Suppl 3):S3-11.

Marchesi S, Lupattelli G, Lombardini R, et al. 2005. Acute inflammatory state during influenza infection and endothelial function. Atherosclerosis, 178:345-50.

Moncada S, Higgs EA. 2006. The discovery of nitric oxide and its role in vascular biology. Br J Pharmacol, 147(Suppl 1):S193-201. 
Roddie IC, Wallace WF. 1979. Methods for the assessment of the effects of drugs on the arterial system in man. Br J Clin Pharmacol, 7:317-23.

Sarabi M, Lind L. 2000. Short-term effects of smoking and nicotine chewing gum on endothelium-dependent vasodilation in young healthy habitual smokers. J Cardiovasc Pharmacol, 35:451-6.

Schmidt AC, Graf C, Brixius K, et al. 2007. Blood pressure-lowering effect of nebivolol in hypertensive patients with type 2 diabetes mellitus: the YESTONO study. Clin Drug Investig, 27:841-9.

Verma S, Szmitko PE, Ridker PM. 2005. C-reactive protein comes of age. Nat Clin Pract Cardiovasc Med, 2:29-36; quiz 58.

Vyssoulis GP, Marinakis AG, Aznaouridis KA, et al. 2004. The impact of third-generation beta-blocker antihypertensive treatment on endothelial function and the prothrombotic state: effects of smoking. Am J Hypertens, 17:582-9.
Whitney RJ. 1953. The measurement of volume changes in human limbs. J Physiol, 121:1-27.

Willerson JT, Ridker PM. 2004. Inflammation as a cardiovascular risk factor. Circulation, 109:II2-10.

Wilson AM, Ryan MC, Boyle AJ. 2006. The novel role of C-reactive protein in cardiovascular disease: risk marker or pathogen. Int $J$ Cardiol, 106:291-7.

Zhang WZ, Venardos K, Chin-Dusting J, et al. 2006. Adverse effects of cigarette smoke on NO bioavailability: role of arginine metabolism and oxidative stress. Hypertension, 48:278-85.

Zoccali C. 2006. Asymmetric dimethylarginine ADMA.: a cardiovascular and renal risk factor on the move. J Hypertens, 24:611-9. 
